\title{
Autoantibody-mediated neurological diseases
}

James A Varley

Sarosh R Irani

James A Varley MB ChB MRCP is a Neurology Clinical Fellow undertaking a DPhil at the University of Oxford, John Radcliffe Hospital, Oxford, UK. He is studying the overlap between autoimmunity and neurodegeneration. Competing interests: none declared.

Sarosh R Irani DPhil FRCP(Neurol) is an Associate Professor and Consultant Neurologist and Wellcome Trust Intermediate Fellow at the University of Oxford, UK, running a laboratory and clinic devoted to autoimmune neurology. Competing interests: SRI is a coapplicant and receives royalties on patent application WO/2010/046716 entitled 'Neurological Autoimmune Disorders'. The patent has been licensed for the development of assays for LGI1 and other VGKC-complexed antibodies. SRI receives associated royalties.

\section{Abstract}

Autoimmune neurological diseases are an expanding group of phenotypically characteristic, potentially treatable syndromes. A number of these conditions are mediated by autoantibodies. The last decade has identified distinctive clinical clues to help diagnose the diseases, definitive investigations and increasing experience regarding the effectiveness of immunotherapies. Awareness of these conditions among nonspecialists - as well as neurologists and psychiatrists - is crucial as early immunotherapy improves outcomes. To discuss the most common antibody-associated neurological conditions, we focus on autoimmune myasthenic syndromes and neuromyotonia in the peripheral nervous system, and their rapidly expanding central nervous system counterparts, which include neuromyelitis optica and autoimmune encephalitis. We mention other related conditions, including stiff person syndrome and classical paraneoplastic neurological syndromes. We note strategies for identification, differential diagnosis, investigation and treatment. Although individually these conditions are uncommon, cumulatively they form a significant group of treatable diseases that frequently present to general medicine and are important for all physicians to recognize promptly.

\section{Keywords}

Autoimmune encephalitis; autoimmune neurological disease; encephalitis; limbic encephalitis; myasthenia gravis; neuromyelitis optica; stiff person syndrome

\section{Key points}

-Autoantibody-mediated neurological diseases are increasingly recognized and diagnosed

- They have distinct clinical phenotypes which can involve the peripheral and/or central nervous systems -Most have a subacute onset, over around 2-6 weeks

-Many have highly sensitive and specific diagnostic autoantibody tests

-Early treatment improves symptoms, improves recovery and decreases mortality; therefore prompt referral and diagnosis is crucial

\section{Introduction}

Neurological diseases with an immune trigger include multiple sclerosis (MS), post-infectious conditions, some forms of epilepsy and encephalitis. In these conditions, data support effector roles for the innate immune system, and for acquired immunity involving $T$ and B-cell populations.

More recently, a group of autoantibody-mediated conditions have increased in importance. The best described is myasthenia gravis (MG). This was followed by the discovery of other autoantibodymediated conditions - most recently involving the central nervous system (CNS). Detection of these 
autoantibodies in the appropriate clinical context often prompts effective immunotherapies. We discuss key clinical features of the most frequently encountered, the differential diagnoses and therapeutic strategies.

\section{Peripheral neurological autoimmune diseases}

\section{Myasthenia gravis}

MG is caused by autoantibodies targeting nicotinic acetylcholine receptors (nAChRs) on the postsynaptic membrane of the neuromuscular junction (NMJ).

Epidemiology: MG occurs with a rate of 5 per million patient-years, although this is rising because of its increased recognition in the elderly. At younger ages, female patients are affected three times more than males versus a male preponderance (2:1) in older age.

Pathogenesis: the two major causative autoantibodies in MG target nAChR and muscle-specific tyrosine kinase (MuSK) (Figure 1). Autoantibodies against the nAChR are predominantly immunoglobulin (Ig) G1 and IgG3 subclasses, which activate complement at the postsynaptic membrane. These antibodies also cause internalization of the nAChR, leading to its reduced surface expression. These effects decrease the ability of the postsynaptic membrane to depolarize, leading to less efficient muscle unit activation and characteristic fatigable weakness. MuSK is also expressed at the postsynaptic membrane of the NMJ. MuSK autoantibodies are predominantly of the lgG4 subclass, which do not activate complement. They prevent MuSK from facilitating clustering of nAChRs, leading to an under-functioning NMJ. ${ }^{1}$

Clinical features: MG typically presents over weeks or months with asymmetrical fatigable weakness in the following muscle groups:

-ocular - producing asymmetrical ptosis and diplopia; ocular symptoms are present in $85 \%$ of patients at presentation, with most progressing to generalized MG within 2 years

-bulbar - causing dysphagia, dysarthria and dysphonia; dysfunction of these muscles can lead to aspiration pneumonia

-limb - proximal and upper limb more commonly than lower limb, and also facial, respiratory and neck weakness.

Symptoms are typically worst at the end of the day and better after rest of the affected muscle group.

\section{Investigations:}

-AChR antibodies - antibodies are positive in around $85 \%$ of patients with clinical features of MG.

-MuSK antibodies - these are positive in around half of the remaining $15 \%$ of patients.

-Electromyography - repetitive stimulation often demonstrates the characteristic 'decrement' of compound muscle action potentials. Single-fibre recordings can demonstrate 'jitter', which represents interpotential variation.

-Tensilon test -administration of edrophonium (an ACh esterase inhibitor) can lead to improvement of cilnical features, and be part of the diagnostic work up. But it is seldom used in modern practice.

- Chest imaging - computed tomography or magnetic resonance imaging (MRI) should be carried out to look for a thymoma (found in 15\%), especially in older patients. Thymic hyperplasia is seen in a further $20 \%$ (usually younger individuals).

\section{Differential diagnosis:}

-Lambert-Eaton myasthenic syndrome (LEMS) - this autoantibody-mediated disease of the presynaptic NMJ often presents with weakness that, in contrast to MG, typically improves after prolonged exercise. LEMS can be clinically distinguished from MG by the presence of autonomic features, reduced tendon reflexes (which become brisker after reinforcement), less frequent ocular involvement and a predilection for the proximal lower limbs. It is associated with small cell lung carcinoma in $50 \%$ of cases, and antibodies to voltage-gated calcium channels in $90 \%$. Electromyography demonstrates reduced compound muscle action potentials, initial decrement and subsequent increment after high-frequency repetitive stimulation. Management involves treatment of the underlying tumour if present, and giving 3,4diaminopyrimidine, a presynaptic potassium channel blocker. Some patients require immunosuppression. | $\quad$ Congenital myasthenia - these are genetic syndromes caused by mutations in NMJ proteins. 
-Amyotrophic lateral sclerosis - this can usually be differentiated by a combination of upper and lower motor neurone features, with prominent muscle wasting and fasciculations.

-Primary muscle disease - myopathies can mimic MG but typically present more symmetrically without ocular involvement.

-Mitochondrial disease - overlaps exist, especially as both have similar ocular features, but mitochondrial disease often has multisystem involvement (e.g. deafness, short stature and diabetes).

\section{Management:}

Assess the severity - particularly of respiratory muscle involvement. A forced vital capacity $<1.5$ litres should prompt referral to intensive care to consider assisted ventilation.

Avoid flare-ups - a myasthenic 'crisis' can be caused by stress, surgery, trauma and infection, and by certain medications including aminoglycosides, macrolides, $\beta$-adrenoceptor blockers, Dpenicillamine, botulinum toxin and fluoroquinolones.

Cholinesterase inhibitors - pyridostigmine is commonly used. It prevents the breakdown of acetylcholine in the NMJ. Dosages are titrated according to symptomatic benefit and adverse effects. The latter are caused by increased parasympathetic tone and include diarrhoea, sweating, excess salivation, constricted pupils and erectile dysfunction. They can be countered with an antimuscarinic agent (propantheline).

Immunosuppression - corticosteroids are used as first-line immunosuppression. These can worsen symptoms over the first 2 weeks if started at high doses, so the dose is often cautiously increased every few days. Typically, after an improvement, corticosteroids are reduced to an effective minimal dosage. A corticosteroid-sparing immunosuppressant such as azathioprine, mycophenolate mofetil or methotrexate is often added from the outset, but corticosteroid-sparing agents can take 3-6 months to produce clinical benefits. Plasma exchange or intravenous Ig (IVIG) are used for rapid clinical response in severe situations, often in the case of bulbar or respiratory involvement.

Thymectomy - this should be carried out in all patients with a thymoma. Thymectomy for thymic hyperplasia is proven to produce an improvement in weakness and reduction in corticosteroid requirements, especially in young patients with nAChR antibodies.

Prognosis: the goal of treatment is to achieve remission of symptoms and minimize the adverse effects of treatment. The clinical course is variable and recovery can be hampered by relapses, but most patients achieve remission with treatment.

\section{Peripheral nerve hyperexcitability (Isaacs' syndrome)}

This condition causes patients to develop continuous, involuntary muscle activity producing cramping, fasciculations and impaired muscle relaxation (pseudomyotonia). Weakness is not usually a feature. The more severe forms of peripheral nerve hyperexcitability are often termed neuromyotonia. Morvan's syndrome describes neuromyotonia in addition to an encephalopathy plus hyperhidrosis and sleep disturbance.

These syndromes are frequently associated with antibodies to contactin-associated protein like 2 (CASPR2) or, more infrequently, leucine-rich glioma inactivated protein 1 (LGI1). Both were previously misattributed as voltage-gated potassium channel (VGKC) antibodies, a test that is now known to have very low syndrome specificity. Measurement of CASPR2 and LGl1 antibodies should be requested in patients with symptoms of neuromyotonia or Morvan's syndrome as there is a good response to immunotherapy. There is a frequent association with tumour, usually a thymoma, and this should be investigated and treated if present.

\section{Central nervous system antibody-associated diseases}

The CNS has traditionally been considered to be an immune-privileged site, but autoantibodies are likely to be pathogenic in an increasing number of CNS diseases. These syndromes often show distinctive clinical features and a good response to immunotherapy. We review the most common ones below.

\section{Myelitis}

Myelitis is inflammation of the spinal cord. It can affect variable longitudinal lengths and transverse areas of the spinal cord. It commonly occurs in the context of MS, infectious diseases and autoantibodymediated diseases. Below, we focus on the latter group. 


\section{Neuromyelitis optica (NMO)}

$\mathrm{NMO}$ is a relapsing inflammatory disease of the CNS characterized by severe optic neuritis and disabling longitudinally extensive transverse myelitis (LETM). NMO has a strong female predominance (9:1). The peak age of onset is 39 years, with an age range from the very young to the very old.

It was previously thought to be a subtype of MS with a more aggressive, disabling course. However, most of the disability in MS arises gradually over several years during the progressive phase of the disease, and most relapses in MS tend to resolve spontaneously. In NMO, the disability arises after severe relapses, and relapse prevention forms the mainstay of management.

In NMO, the myelitis typically involves the cord over more than three vertebral segments ('longitudinally extensive'), and the optic neuritis is often severe and sometimes bilateral. Patients can also have optic neuritis with LETM, isolated LETM, isolated optic neuritis and other increasingly recognized variants of the more classical syndrome. NMO shows a poor, often adverse, response to the drugs used to treat MS, such as interferon- $\beta$ and natalizumab.

The discovery of antibodies to aquaporin 4 (AQP4), a water transport channel present in astrocyte foot processes, recharacterized NMOSD as a primarily antibody-mediated disorder. AQP4antibodies are found in $90 \%$ of patients with classical NMO with optical neuritis, and $50 \%$ of those with more atypical presentations. Many patients without AQP4-antibodies have antibodies to myelin oligodendrocyte glycoprotein (MOG). These patients usually have a monophasic disease course akin to Devic's original description, with bilateral optic nerve disease and involvement at the conus of the spine. Some patients with MOG antibodies have paediatric onset acute disseminated encephalomyelitis.

After excluding infectious and metabolic causes, pulsed intravenous methylprednisolone is given, with plasma exchange or IVIG in those severely affected. Long-term immunomodulation, frequently with corticosteroid-sparing agents, follows in most patients with AQP4 antibodies as the relapse rate is high. There is a $60-80 \%$ reduction in relapses in patients taking azathioprine, mycophenolate mofetil and rituximab. ${ }^{2}$ Symptomatic therapies are important, especially as troublesome neuropathic pain is frequent.

\section{Autoimmune encephalitis}

Encephalitis is parenchymal brain inflammation and traditionally associated with viral infection, particularly herpes simplex virus. Autoimmune aetiologies are now recognized as a more common cause of encephalitis than infections. The two most common of the neuronal surface autoantibodies associated autoimmune encephalitis are discussed below33 (Table 1, Figure 2), with rarer causes shown in Table 2.

\section{Limbic encephalitis (LE)}

In LE, inflammation centres on the medial temporal lobes (Figure 3a) and the clinical syndrome includes amnesia, disorientation and seizures. This is most commonly associated with antibodies to LGI1.

LGI1 antibody LE: the most common specific autoantibody target in patients with LE is LGl1, which usually causes a non-paraneoplastic LE ( $<5 \%$ rate of tumours) affecting males more than females $(2: 1)$ at a median age of 68 years. Onset is usually over days or weeks with a variety of seizure types, anterograde and retrograde memory impairment, disorientation and personality change. Serum hyponatraemia occurs in 50\%, and in $60 \% \mathrm{MRI}$ brain scans reveal medial temporal lobe T2 hyperintensities (Figure 3a). Both MRI and cerebrospinal fluid (CSF) results can be normal. $>90 \%$ of patients carry the HLADRB $1{ }^{*} 07: 01$ allele.

Patients may have typical mesial temporal lobe seizures or characteristic 'faciobrachial dystonic seizures' (FBDS). These high-frequency (50-100 a day), brief ( $<3$ seconds) seizures involving ipsilateral arm and face (Figure $3 b$ ) are usually resistant to antiepileptic drugs but improve with immunotherapy. Patients with isolated FBDS usually progress to LE, and prompt immunotherapies may prevent this progression and the resultant cognitive impairment.

Less common forms of autoantibody-mediated LE can be caused by autoantibodies against CASPR2 and the $y$-aminobutyric acid (GABA) A and B receptors. ${ }^{4}$

$\mathrm{N}$-methyl-D-aspartate receptor (NMDAR) antibody encephalitis NMDAR antibodies present with a more diffuse encephalitis. 
Epidemiology: this occurs with an incidence of around 1-2 per million per year, predominantly affecting young female patients, with a peak age of onset at 21 years.

Pathophysiology: this condition is caused by NMDAR antibodies that cross-link and internalize NMDARs in the CNS. There is infiltration of $B, T$ and plasma cells in the brain; the latter are probably responsible for local NMDAR IgG secretion. In around one-third of patients, the disease is associated with an ovarian teratoma that expresses NMDARs. The teratoma is the likely immunizing agent, leading to a breakdown in immunological tolerance. NMDAR antibody encephalitis can also occur a few weeks after a proven viral encephalitis, typically herpes simplex viral encephalitis (HSVE), and autoimmune encephalitis should be considered in 'relapses' after HSVE.

\section{Investigations:}

-NMDAR antibodies - these are best tested from paired serum and CSF, allowing estimation of the relative amount of 'intrathecal' antibody synthesis.

-Electroencephalography - this is abnormal in most patients and can be useful to monitor for overt seizure activity. It occasionally shows a characteristic 'extreme delta brush' pattern.

-Lumbar puncture - lymphocytosis is seen in two-thirds of cases. Oligoclonal bands are present in around half of patients and often late in the disease course.

-MRI of the brain - this is normal in around $80 \%$. In some, white matter or hippocampal T2 hyperintensities are demonstrated.

-Pelvic MRI - this identifies associated ovarian teratomas, most prevalent in individuals aged 16-35 years, which can be very small and require detailed imaging.

Clinical features: symptom progression is usually highly stereotyped (see Table 1), often beginning with a short prodrome of headache, fever and flu-like symptoms.

-Early (first 2-3 weeks) - initially, a transdiagnostic psychiatric presentation with delusions, agitation, anxiety, mood lability, aggression and hallucinations. This often takes patients to inpatient mental health services. Neurological features emerge and include cognitive impairment, dysphasia and seizures. -Late (after week 3) - complex hyperkinetic movement disorder with autonomic dysfunction and progression to coma in severe cases. Also, catatonia or Parkinsonian features can be present. There is variable severity, and many patients do not require intensive care support.

Management: with immunotherapy and supportive measures, patients typically recover albeit slowly, over several weeks to months. However, some patients who make a good final recovery may require hospitalization for 2 years. Early diagnosis and immunotherapy improve outcomes.

Half of patients respond to first-line treatment including pulsed intravenous methylprednisolone, with or without plasma exchange and IVIG. Most non-responders improve after second-line treatment with rituximab or cyclophosphamide. Prompt removal of ovarian teratomas improves outcomes. Symptomatic medications may be needed for the movement disorder, agitation and psychosis..

Prognosis: mortality is $10 \%$ with an approximately $10 \%$ relapse rate ${ }^{5}$ Relapses appear to be preventable with immunotherapy.

By contrast to the diseases discussed above, those below are associated with autoantibodies that target intracellular epitopes. These antibodies are not considered directly pathogenic, but markers of an underlying disease.

\section{Glutamic acid decarboxylase (GAD)}

GAD is an intracellular enzyme that catalyses a key step in the production of GABA, a major inhibitory neurotransmitter in the CNS. Autoantibodies to GAD are associated with type 1 diabetes mellitus (at low concentrations), and higher concentrations with autoimmune LE and stiff person syndrome (SPS).

LE associated with GAD-antibodies occurs in young women and has a longer history, poor recovery and limited response to immunotherapy, in contrast to LGl1-antibody LE. 


\section{Stiff person syndrome}

SPS is characterized by insidious paraspinal or lower limb rigidity sometimes spreading to the abdomen, arms and face. Muscle spasms with a startle response can be triggered by sound and touch. A diagnostic delay of years is frequent. The cause is immune-mediated impairment of GABAergic interneurones in the brain and spinal cord, initiating aberrant muscular contraction. However, the final effectors are not identified, as yet. There is progressive disability, with most patients needing an ambulatory aid. Management is with GABA augmentation, using either benzodiazepines or baclofen, often with immunotherapy, typically IVIG.

\section{Paraneoplastic autoantibody-associated neurological diseases}

Neuronal autoantibodies which target surface-expressed antigens (including the NMDAR, see Table 2 for information on CASPR2, the a-amino-3-hydroxy-5-methyl-4-isoxazolepropionic acid (AMPA) receptor and $\mathrm{GABA}_{\mathrm{B}}$ antibodies) may be associated with cancer and respond well to immunotherapy alongside appropriate oncological treatment.

'Classical' paraneoplastic (onconeuronal) autoantibodies target intracellular antigens and are almost always associated with an underlying cancer. These have a predominantly T-cell-driven immune response, a poor prognosis and little response to immunotherapy.

The main antigens and their common syndromes include:

- Hu - encephalomyelitis and sensory peripheral neuropathy; most often seen with small cell lung cancer -Yo - cerebellar syndrome; associated with breast and ovarian cancer

- Ri - cerebellar syndrome, opsoclonus; associated with ovarian and endometrial cancer

- CV2 - encephalomyelitis with sensory peripheral neuropathy; associated with thymoma and small cell lung cancer

-Ma2 - LE, SPS and cerebellar syndrome; associated with testicular tumours and one 'onconeural' antibody syndrome that shows a response to immunotherapies. 


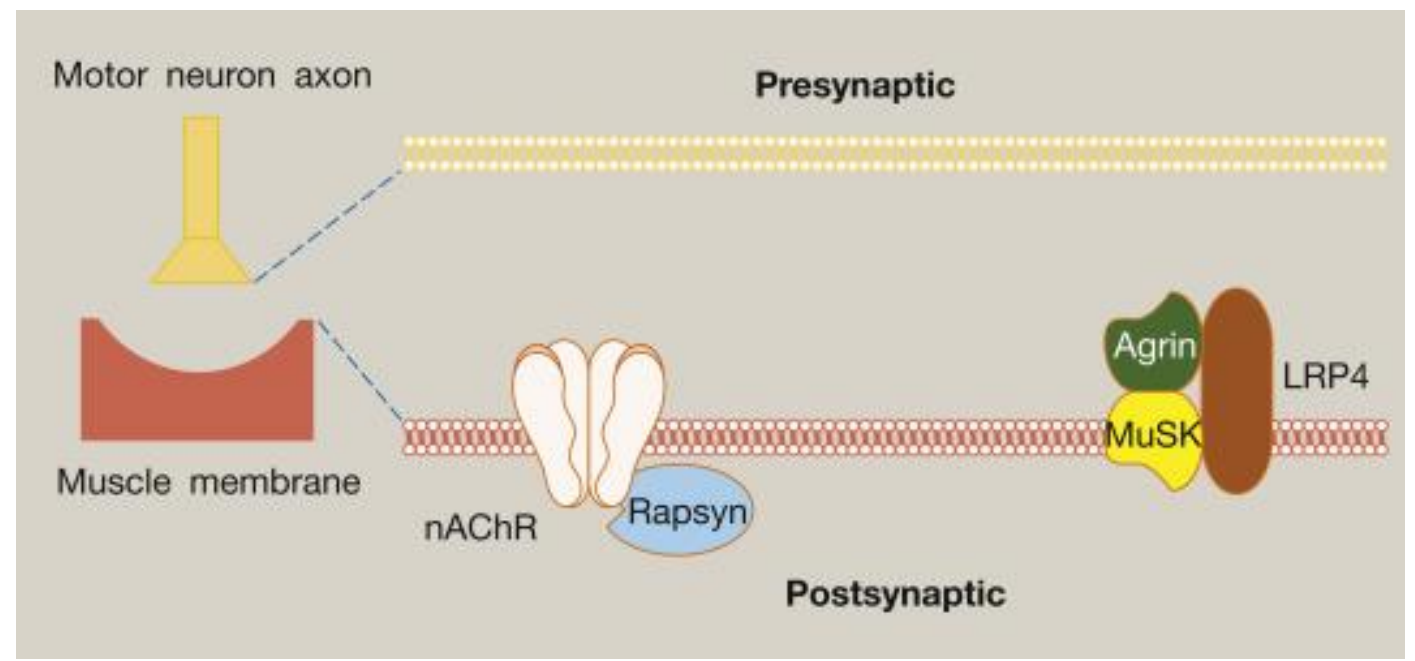

Figure 1. Antigenic targets and key proteins at the NMJ. The key components include the nAChRs, which require rapsyn for clustering. Another membrane-associated complex also plays a key role in receptor clustering and includes MuSK, another autoantibody target in MG. MuSK associates with agrin and lowdensity lipoprotein receptor-related protein 4 (LRP4). 


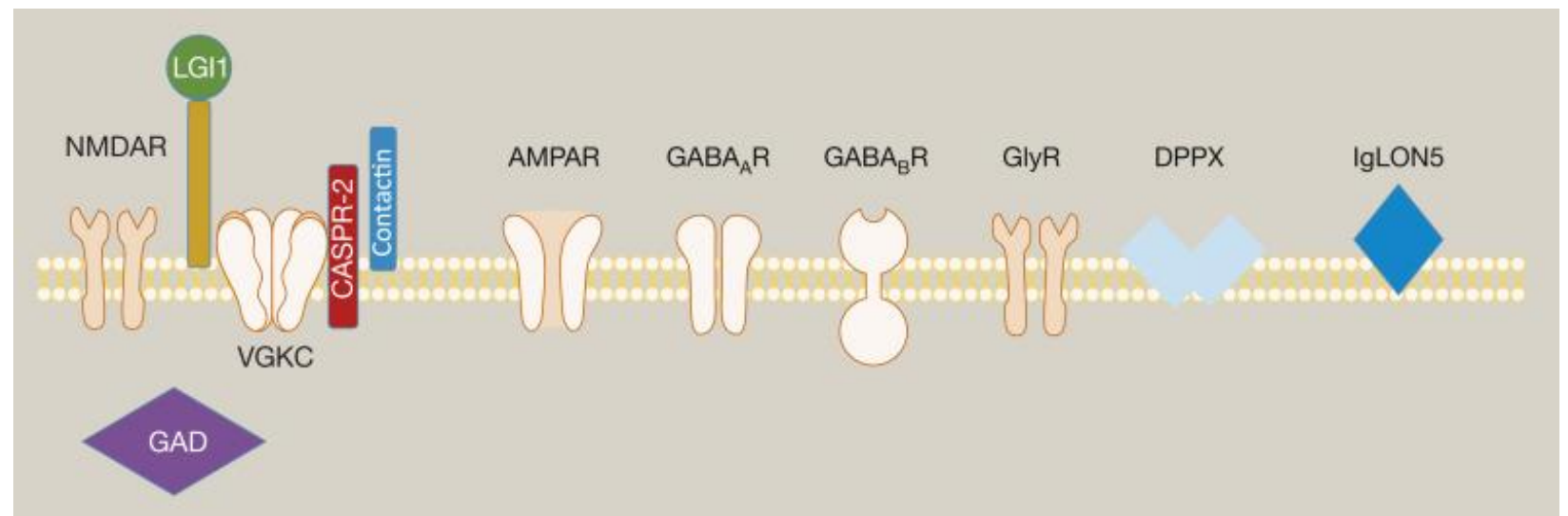

Figure 2. The most common extracellular and intracellular autoantibody targets associated with CNS diseases. Shapes are based on the three-dimensional crystal structure of the protein. These include NMDAR ( $N$-methyl-D-aspartate receptor), AMPAR ( $\alpha$-amino-3-hydroxy-5-methyl-4-isoxazolepropionic acid receptor), LGI1 (leucine-rich glioma-inactivated protein 1), CASPR2 (contactin-associated protein-like 2), GAD (glutamic acid decarboxylase), GABA ${ }_{A}$ or GABA $(\gamma$-aminobutyric acid) receptors, GlyR (glycine receptor), DPPX (dipeptidyl-peptidase-like protein 6) and IgLON5 (Ig-like domain-containing protein 5). 


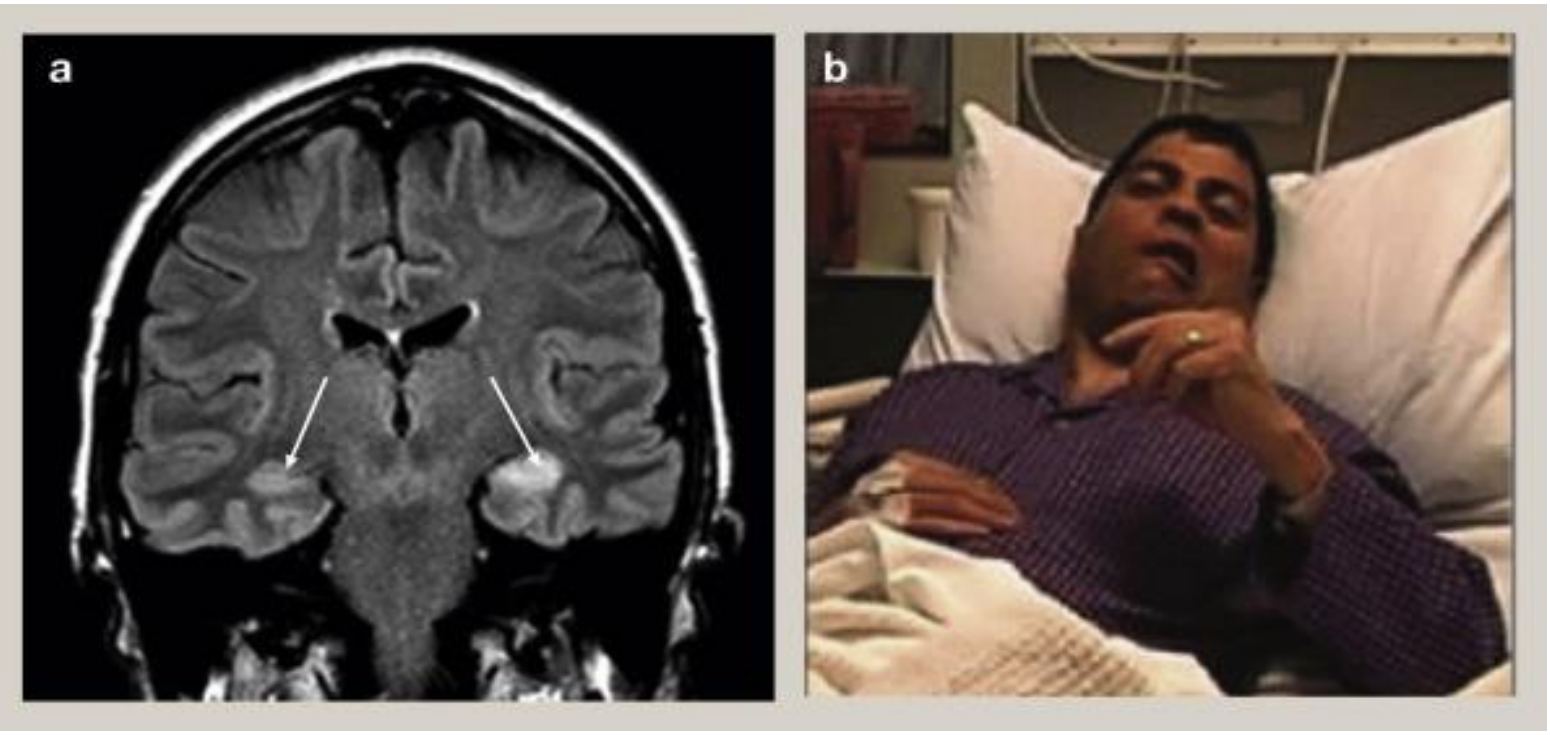

Figure 3. Features of limbic encephalitis. (a) T2-weighted MRI scan showing characteristic high signal in the medial temporal lobes (arrows) associated with limbic encephalitis. (b) A patient having a faciobrachial dystonic seizure, with brief left-sided facial and ipsilateral upper limb dystonic episodes.

Reproduced with permission from Irani SR, Mitchell AW, Lang B, et al. Faciobrachial dystonic seizures precede Lgi1 antibody limbic encephalitis. Ann Neurol 2011; 69: 892-900. 


\begin{tabular}{|c|c|c|c|c|c|}
\hline Antigen & Typical clinical features & $\begin{array}{l}\text { Cerebrospinal } \\
\text { fluid }\end{array}$ & MRI & Management & Prognosis \\
\hline NMDAR & $\begin{array}{l}\text { Non-specific viral prodrome } \\
\text { Early - psychiatric features, cognitive } \\
\text { dysfunction, seizures } \\
\text { Late - movement disorder, coma and } \\
\text { autonomic dysfunction }\end{array}$ & $\begin{array}{l}60 \% \\
\text { lymphocytosis }\end{array}$ & Often normal & $\begin{array}{l}\text { First-line: CS and } \\
\text { PLEX } \pm \text { IVIG } \\
\text { Second-line: } \\
\text { CYP/rituximab }\end{array}$ & $\begin{array}{l}80 \% \text { respond to either } \\
\text { first- or additional } \\
\text { second-line treatment to } \\
\text { become functionally } \\
\text { independent }\end{array}$ \\
\hline LGl1 & $\begin{array}{l}\text { Early FBDS - frequent, very brief } \\
\text { dystonic seizures affecting the face } \\
\text { and arms, along with other seizure } \\
\text { types } \\
\text { Late - LE: triad of seizures, memory } \\
\text { loss and disorientation. Can occur with } \\
\text { or without FBDS }\end{array}$ & Usually normal & $\begin{array}{l}\text { Medial temporal lobe } \\
\text { and basal ganglia T2 } \\
\text { hyperintensities } \\
\text { seen in about } 60 \%\end{array}$ & $\begin{array}{l}\text { Oral/intravenous } \\
\text { steroids and } \\
\text { frequent escalation } \\
\text { to PLEX/IVIG }\end{array}$ & $\begin{array}{l}\text { Overall good, typically } \\
\text { with residual memory } \\
\text { deficit. Relapses } \\
\text { possible, especially with } \\
\text { limited immunotherapy }\end{array}$ \\
\hline CASPR2 & $\begin{array}{l}\text { Peripheral nerve } \\
\text { hyperexcitability/neuromyotonia } \\
\text { Morvan's syndrome - neuromyotonia, } \\
\text { pain, hyperhidrosis, severe insomnia } \\
\text { and hallucinations; LE; isolated } \\
\text { cerebellitis }\end{array}$ & Usually normal & $\begin{array}{l}\text { Normal in around } \\
70 \% \text {, depending on } \\
\text { clinical syndrome }\end{array}$ & As above & $\begin{array}{l}\text { Good response to } \\
\text { immunotherapies if no } \\
\text { thymoma present }\end{array}$ \\
\hline GAD & $\begin{array}{l}\text { SPS } \\
\text { Rarely LE }\end{array}$ & $\begin{array}{l}\text { Can detect } \\
\text { oligoclonal } \\
\text { bands in SPS }\end{array}$ & $\begin{array}{l}\text { Normal in SPS, } \\
\text { often medial } \\
\text { temporal lobe } \\
\text { hyperintensities in } \\
\text { LE }\end{array}$ & $\begin{array}{l}\text { SPS - BDZ and } \\
\text { baclofen, IVIG } \\
\text { CS in LE }\end{array}$ & $\begin{array}{l}\text { Moderate reported } \\
\text { response to } \\
\text { immunotherapies }\end{array}$ \\
\hline
\end{tabular}

BDZ, benzodiazepines; CS, corticosteroids; CYP, cyclophosphamide; PLEX, plasma exchange. For other abbreviations, see text.

Table 1. 
List of rarer antibody-mediated encephalitides with key clinical features

\begin{tabular}{|l|l|}
\hline $\begin{array}{l}\text { Antigen } \\
\text { AMPA } \\
\text { receptor }\end{array}$ & $\begin{array}{l}\text { Key clinical features } \\
\text { Primarily affects elderly individuals, with a female predominance; 70\% are associated with } \\
\text { malignancy (SCLC, breast carcinoma, thymoma). LE with a high mortality but a good } \\
\text { response to treatment and oncological therapy }\end{array}$ \\
\hline $\begin{array}{l}\text { GABA } A \\
\text { receptor }\end{array}$ & $\begin{array}{l}\text { Affects the young, presents with seizures and psychiatric/cognitive disturbances, } \\
\text { sometimes status epilepticus that can be refractory to conventional management. Around } \\
80 \% \text { response to immunotherapy, with a higher mortality associated with treatment- } \\
\text { resistant status epilepticus }\end{array}$ \\
\hline $\begin{array}{l}\text { GABA } \\
\text { receptor }\end{array}$ & $\begin{array}{l}\text { Elderly, LE with prominent seizures, 50\% association with SCLC. Often a good response } \\
\text { to immunotherapy and oncological treatment }\end{array}$ \\
\hline $\begin{array}{l}\text { Glycine } \\
\text { receptor }\end{array}$ & $\begin{array}{l}\text { Stiff person-like disease - often its most severe phenotype, PERM. A better response to } \\
\text { immunotherapy than anti-GAD-associated SPS }\end{array}$ \\
\hline DPPX & $\begin{array}{l}\text { Encephalitis with tremor and myoclonus. Associated severe, non-infective diarrhoea or } \\
\text { constipation resulting from expression of antigen in the enteric nervous system. Good } \\
\text { response to immunotherapy, no clear tumour association. High relapse rate on withdrawal } \\
\text { of immunotherapy }\end{array}$ \\
\hline IgLON5 & $\begin{array}{l}\text { Neurodegenerative disorder with parasomnia associated with non-rapid and rapid eye } \\
\text { movement sleep as well as disordered breathing and an associated movement disorder. } \\
\text { No response to immunotherapy }\end{array}$ \\
\hline $\begin{array}{l}\text { DPPX, dipeptidyl-peptidase-like protein 6; IgLON5, immunoglobulin-like domain-containing protein 5; PERM, progressive } \\
\text { encephalopathy with rigidity and myoclonus; SCLC, small cell lung cancer. For other abbreviations, see text. }\end{array}$
\end{tabular}

Table 2. 


\section{KEY REFERENCES}

1 Binks S, Vincent A, Palace J. Myasthenia gravis: a clinical immunological update. J Neurol 2016; 263: 826-34.

2 Trebst C, Jarius S, Berthele A, et al. Update on the diagnosis and treatment of neuromyelitis optica: recommendations of the Neuromyelitis Optica Study Group (NEMOS). J Neurol 2014; 261: 1-16. 3 Irani SR, Gelfand JM, Al-Diwani A, Vincent A. Cell-surface central nervous system autoantibodies: clinical relevance and emerging paradigms. Ann Neurol 2014; 76: 168-84.

4 Varley J, Vincent A, Irani SR. Clinical and experimental studies of potentially pathogenic brain-directed autoantibodies: current knowledge and future directions. J Neurol 2014; 262: 1081-95.

5 Titulaer MJ, McCracken L, Gabilondo I, et al. Treatment and prognostic factors for long-term outcome in patients with anti-NMDA receptor encephalitis: an observational cohort study. Lancet Neurol 2013; 12: 157-65.

\section{FURTHER READING \\ Hoftberger R, Rosenfeld MR, Dalmau J. Update on neurological paraneoplastic syndromes. Curr Opin Oncol 2015; 27: 489-95.}

Irani SR, Alexander S, Waters $\mathrm{P}$, et al. Antibodies to Kv1 potassium channel-complex proteins leucinerich, glioma inactivated 1 protein and contactin-associated protein-2 in limbic encephalitis, Morvan's syndrome and acquired neuromyotonia. Brain 2010; 133: 2734-48.

Irani SR, Michell AW, Lang B, et al. Faciobrachial dystonic seizures precede Lgi1 antibody limbic encephalitis. Ann Neurol 2011; 69:892-900.

Saiz A, Blanco Y, Sabater L, et al. Spectrum of neurological syndromes associated with glutamic acid decarboxylase antibodies: diagnostic clues for this association. Brain 2008; 131: 2553-63.

Thompson J, Bi M, Murchison AG, Makuch M, et al., and the FBDS study group. The importance of early immunotherapy in 103 patients with faciobrachial dystonic seizures. Brain 2018; 141: 348-56. 